\title{
AHP and TOPSIS methods applied in the field of scientific research
}

\author{
El Mohadab Mohamed ${ }^{1}$, Belaid Bouikhalene ${ }^{2}$, Fahd Ouatik ${ }^{3}$, Said Safi ${ }^{4}$ \\ ${ }^{1,2,4}$ Department of Mathematics and Computer Science, Sultan Moulay Slimane University, Morocco \\ ${ }^{3}$ Department of Physics, Cadi Ayyad University, Morocco
}

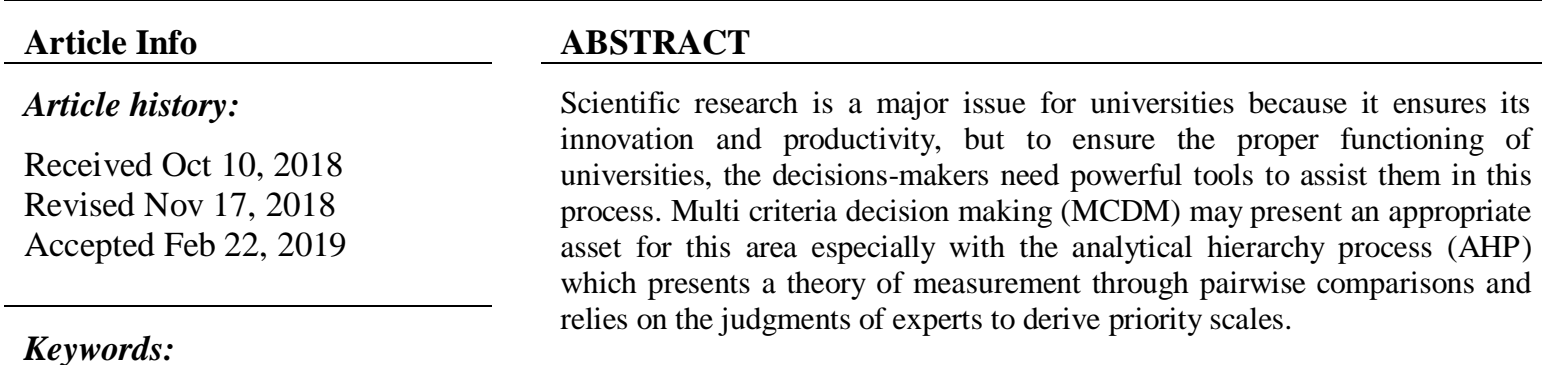

Decision making

Multi criteria decision making

AHP

Scientific research

TOPSIS

Copyright $₫ 2019$ Institute of Advanced Engineering and Science. All rights reserved.

\section{Corresponding Author:}

El Mohadab Mohamed,

Department of Mathematics and Computer Science,

Sultan Moulay Slimane University, Mghila BP: 592 Beni Mellal,

PB: 592 Mghila, Beni Mellal, Morocco.

Email:m.elmohadab@gmail.com

\section{INTRODUCTION}

The good governance [1] requires the modernization and rationalization of management information decision assist system in administrative and managerial aspects. The management and automation of scientific research in universities [2] represents a great challenge for universities especially for the decisionsmakers which comes the need of find techniques and solutions suitable for their specific purposes. The decision making system [3] is a process based on best practices related to the university's strategy that deal with complex evaluation, prioritization, and selection situation. Because not all information is useful, and a lot of information cannot guarantee fthat we understand better the decisions-makers need to determine: the problem, the aim and objective from the decision, the criteria of the decision, and the consequences of this decision.

In this direction Analytic Hierarchy Process (AHP) represent one of the methods of Multi Criteria Decision Making (MCDM) that's usually used to solve some problem that heavily involves human participation and judgments. The paper has three parts. First section describes the different research methods. Full Research methodology and results are reported in the second section before concluding.

\section{RESEARCH METHOD}

\subsection{Multiple Criteria Decision Making}

Multiple criteria decision-making [4] (MCDM) are used in order to solve problems related to several criteria. Multiple criteria decision-making are regrouped into two sections: 
Firstly, multi-attribute decision making [5] (MADM) this method is used to solve problems with discrete decision spaces and a predetermined or a limited number of alternative choices, is related to the judgment of the personal statement like the choice of (new managers, the choice of new provider...) Between the popular technique we find Analytic Hierarchy Process (AHP), ANP, TOPSIS, ELECTRE, MAUT, and PROMETHEE I \& II.

Secondly multi-objective decision making (MODM) [6] this method is used when we have decision variable values that are determined in a continuous or integer domain with either an infinitive or a large number of alternative choices, the best of which should satisfy the decision-maker constraints and preference priorities. Between the popular techniques we find fuzzy analytic hierarchy process (FAHP).

\subsection{Techniques in Multiple Criteria Decision Making}

The main steps followed in multiple criteria decision-making:

1) Define the Problem by specifying the object that must be realistic and measurable.

2) Determine the requirement.

3) Establish the goals

4) Identify the alternative.

5) Develop evaluation criteria.

6) Selecting decision making tool.

7) Apply the tool.

8) Find the result.

For the selection of the criteria that must be [7]:

1) Able to distinguish among alternatives.

2) Complete enough to cover all goals.

3) Non-redundant.

4) Few numbers.

5) Operational and meaningful.

\subsection{Analytic Hierarchy Process Principles [8]}

Discovering by Saaty AHP can be combined with another technique like Fuzzy logic, linear programming to provide a better result in their areas. The use of AHP is due to the steps imposed by the seven techniques [9]:

1) Define the Problem

2) Determine the objectives and expected results.

3) Determine the main criteria involved.

4) Prioritize the problem in different levels, Let $\mathrm{D}$ is a $\mathrm{n} \mathrm{x}$ pair-wise comparison matrix.

$$
D=\left[\begin{array}{cccc}
\mathrm{a}_{11} & \mathrm{a}_{21} & \ldots & \mathrm{a}_{1 \mathrm{n}} \\
a_{21} & a_{22} & \ldots & a_{2 n} \\
\vdots & \vdots & \ddots & \vdots \\
a_{n 1} & a_{n 2} & \ldots & a_{n n}
\end{array}\right]
$$

Diagonal elements are all equal to 1.

5) Compare each element in the corresponding level, by normalizing the matrix with geometric means, where:

$$
\begin{gathered}
w_{i}=\frac{\left[\sum_{j=1}^{n} a_{i j}\right]^{1 / n}}{\sum_{i=1}^{n}\left[\sum_{j=1}^{n} a_{i j}\right]^{1 / n}} \\
\mathrm{i}, \mathrm{j}=1,2, \ldots \ldots . \mathrm{n}
\end{gathered}
$$

Perform consistency check. If $\mathrm{C}$ denotes $\mathrm{n}$ dimensional column vector describing the sum of:

$$
\mathrm{C}=\left[C_{i}\right]_{\mathrm{nx} 1}=D \mathrm{~W}^{\mathrm{T}}
$$

where $\mathrm{i}=1,2, \ldots \ldots$. 
$\left[w_{1} w_{2} \ldots \ldots w_{n}\right]=\left[\begin{array}{l}C_{1} \\ C_{2} \\ C_{n}\end{array}\right] D \mathrm{~W}^{\mathrm{T}}=\left[\begin{array}{cccc}1 & a_{12} \ldots \ldots & a_{1 n} \\ a_{21} & 1 \ldots \ldots & a_{2 n} \\ a_{n 1} & a_{n 2} \ldots \ldots & 1\end{array}\right]$

6) Find the maximum eigenvalue, consistency ratio (CR), consistency index (CI).

$$
\begin{aligned}
& \lambda_{\max }=\frac{\sum_{i=1}^{n} c v_{i}}{n} \\
& \mathrm{CI}=\frac{\lambda_{\max -n}}{n-1} \\
& \mathrm{CR}=\frac{C l}{R l}
\end{aligned}
$$$$
\text { Where } \mathrm{i}=1,2, \ldots \ldots . \mathrm{n}
$$

Where RI denotes average random index.

7) Repeat the operation until you reach the values in the desired range.

\subsection{Technique for Order Preference by Similarity to Ideal Solution}

Technique for Order Preference by Similarity to Ideal Solution (TOPSIS) [10] has been used in a various comparisons of alternatives such: ranking leaders or entities item selection from among alternatives, supply chain operations, data mining, etc.

TOPSIS can be summarized is that the selected alternative should have shortest distance, in a geometrical sense, from the ideal solution and longest distance from the worst solution. It is one of the classical MCDM approach, based on aggregating function to find a solution which is nearest to positive ideal solution and farthest from negative ideal solution.

\section{RESULTS AND ANALYSIS}

In this paper, we choose to work with the dataset provided by laboratories which belongs to the Sultan Moulay Slimane University. also we choose to apply multi-attribute decision making method through the analytic hierarchy process (AHP) technique by choosing the adequate dimensions. In this order the decision makers want to know the laboratory with the highest score the last year, the decision will be based on four factors:

a. The number of new registered in the laboratory the previous year.

b. The number of publications in the laboratory the previous year.

c. The number of thesis supported in the laboratory the previous year.

d. The number of events organized by the laboratory the previous year.

Decision-makers consider the number of publications from members of each research laboratory as the most important factor in the decision, and give less importance to the new doctoral candidate in the first year.

1) The number of publications in the previous year.

2) The number of thesis supported in the previous year.

3) The number of events organized by the laboratory in the previous year.

4) The number of new registered in the laboratory in the previous year.

According to the data collected from the scientific research service, the study is limited to five research laboratories:

\begin{tabular}{cccccc}
\hline Variable & MPA & SL & ES & MET & RCC \\
\hline Publications & 10 & 16 & 11 & 12 & 8 \\
Thesis & 2 & 4 & 1 & 2 & 1 \\
Publications & 3 & 5 & 4 & 1 & 2 \\
New-Registered & 12 & 11 & 13 & 10 & 14 \\
\hline
\end{tabular}

\footnotetext{
a. MPA: Mathématique Physique Appliquée.

b. SL: Sciences Langage.

c. ES: Environnement Santé.

d. MET: Modélisation des Ecoulements des Transferts.

e. RCC: Recherche Culture Communication.
} 


\subsection{AHP}

Step1: we create a diagram based on the objectives of the decision criteria and alternative solutions:

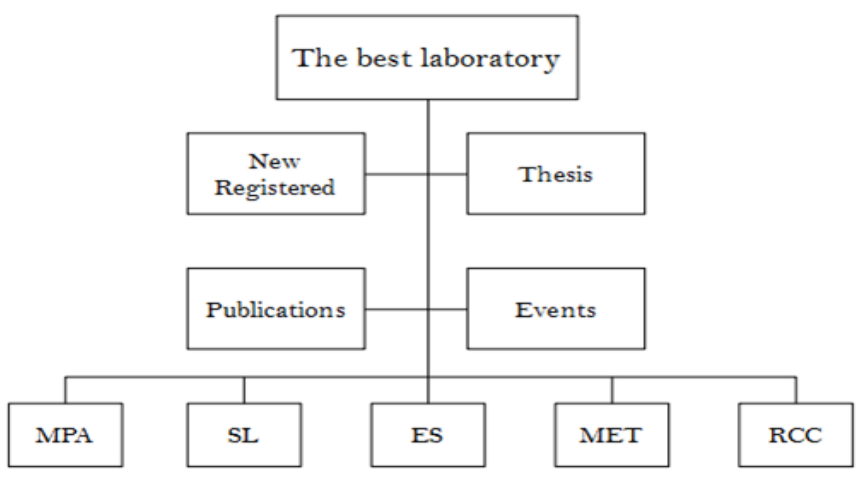

Figure 1. Diagram of the decision criteria and alternative solutions

Step2: A decision criterion matrix is made in order to compare them:

\begin{tabular}{ccccc}
\hline & Events & Publications & New-Registered & Thesis \\
\hline Events & 1 & $1 / 4$ & 2 & $1 / 2$ \\
Publications & 4 & 1 & 1 & 2 \\
New-Registered & $1 / 2$ & $1 / 5$ & 3 & $1 / 3$ \\
Thesis & 2 & $1 / 2$ & 1 & 1 \\
\hline
\end{tabular}

The importance of each criterion with respect to another according to the following scale:

$1=$ Equal importance.

2 = Moderate importance.

$3=$ Strong importance.

$4=$ very strong importance.

$5=$ extreme importance.

Ratio $=$ importance of the criterion on the line / importance of the criterion on the column.

A second matrix to express each ratio in relative percentage:

\begin{tabular}{|c|c|c|c|c|c|}
\hline & Events & Publications & New-Registered & Thesis & average \\
\hline Events & 0.133 & 0.128 & 0.182 & 0.130 & 0.143 \\
\hline Publications & 0.533 & 0.513 & 0.455 & 0.522 & 0.506 \\
\hline New-Registered & 0.067 & 0.103 & 0.091 & 0.087 & 0.087 \\
\hline Thesis & 0.267 & 0.256 & 0.0273 & 0.261 & 0.264 \\
\hline
\end{tabular}

Step3: For each criterion of decision, a matrix is created which makes it possible to compare the different solutions:

Events

\begin{tabular}{lccccc}
\hline & MPA & SL & ES & MET & $1 / 3$ \\
MPA & 1 & 3 & 2 & $1 / 5$ & $1 / 2$ \\
SL & $1 / 3$ & 1 & $1 / 2$ & $1 / 5$ & $1 / 4$ \\
ES & $1 / 2$ & 2 & 1 & 1 & $1 / 4$ \\
MET & 3 & 5 & 5 & $1 / 2$ & 1 \\
RCC & 2 & 4 & 4 & & 1 \\
\hline
\end{tabular}

Matrix with relative percentages:

\begin{tabular}{lcccccc}
\multicolumn{7}{c}{ Matrix with relative percentages: } \\
\hline MPA & SL & ES & MET & RCC \\
\hline MPA & 0.146 & 0.2 & 0.16 & 0.149 & 0.125 & 0.062 \\
SL & 0.048 & 0.066 & 0.04 & 0.089 & 0.089 & 0.062 \\
ES & 0.073 & 0.133 & 0.08 & 0.447 & 0.5 & 0.087 \\
MET & 0.439 & 0.333 & 0.4 & 0.223 & 0.423 & 0.25 \\
RCC & 0.292 & 0.266 & 0.32 & & 0.272 \\
\hline
\end{tabular}


Publications

\begin{tabular}{|c|c|c|c|c|c|}
\hline & MPA & SL & ES & MET & RCC \\
\hline MPA & 1 & 4 & 2 & 3 & $1 / 2$ \\
\hline SL & $1 / 4$ & 1 & $1 / 3$ & $1 / 2$ & $1 / 5$ \\
\hline ES & $1 / 2$ & 3 & 1 & 2 & $1 / 3$ \\
\hline MET & $1 / 3$ & 2 & $1 / 2$ & 1 & $1 / 4$ \\
\hline RCC & 2 & 5 & 3 & 4 & 1 \\
\hline
\end{tabular}

Matrix with relative percentages:

\begin{tabular}{lcccccc}
\hline & MPA & SL & ES & MET & RCC & average \\
\hline MPA & 0.245 & 0.266 & 0.292 & 0.285 & 0.219 & 0.261 \\
SL & 0.061 & 0.066 & 0.048 & 0.047 & 0.087 & 0.061 \\
ES & 0.122 & 0.2 & 0.146 & 0.190 & 0.141 & 0.160 \\
MET & 0.080 & 0.133 & 0.73 & 0.095 & 0.098 \\
RCC & 0.490 & 0.333 & 0.439 & 0.380 & 0.438 \\
\hline
\end{tabular}

New-Registered

\begin{tabular}{lccccc}
\hline & MPA & SL & ES & MET & $1 / 3$ \\
MPA & 1 & 3 & 2 & $1 / 5$ & $1 / 2$ \\
SL & $1 / 3$ & 1 & $1 / 2$ & $1 / 4$ & $1 / 4$ \\
ES & $1 / 2$ & 2 & 1 & 4 & $1 / 3$ \\
MET & 3 & 5 & 3 & $1 / 2$ & 1 \\
RCC & 2 & 4 & & & 1 \\
\hline
\end{tabular}

Matrix with relative percentages:

\begin{tabular}{lcccccc}
\hline & MPA & SL & ES & MET & RCC & average \\
\hline MPA & 0.146 & 0.2 & 0.190 & 0.144 & 0.122 & 0.160 \\
SL & 0.048 & 0.066 & 0.047 & 0.087 & 0.061 & 0.172 \\
ES & 0.073 & 0.133 & 0.095 & 0.109 & 0.080 & 0.490 \\
MET & 0.439 & 0.333 & 0.380 & 0.438 & 0.219 & 0.245 \\
RCC & 0.292 & 0.266 & 0.285 & & 0.261 \\
\hline
\end{tabular}

Thesis

\begin{tabular}{lccccc}
\hline & MPA & SL & ES & MET & $1 / 3$ \\
MPA & 1 & $1 / 2$ & 2 & $1 / 2$ & 3 \\
SL & 2 & 1 & 3 & $1 / 4$ & 3 \\
ES & $1 / 2$ & $1 / 3$ & 1 & 1 & 2 \\
MET & 3 & 2 & 4 & 5 & $1 / 5$ \\
RCC & $1 / 3$ & $1 / 3$ & $1 / 2$ & 1 \\
\hline
\end{tabular}

Matrix with relative percentages:

\begin{tabular}{lcccccc}
\hline & MPA & SL & ES & MET & RCC \\
\hline MPA & 0.146 & 0.120 & 0.190 & 0.144 & 0.214 & 0.162 \\
SL & 0.292 & 0.240 & 0.285 & 0.219 & 0.214 & 0.250 \\
ES & 0.073 & 0.079 & 0.095 & 0.109 & 0.142 & 0.099 \\
MET & 0.439 & 0.480 & 0.380 & 0.438 & 0.357 & 0.418 \\
RCC & 0.048 & 0.079 & 0.047 & 0.087 & 0.071 & 0.066 \\
\hline
\end{tabular}

Step4: We will create a solution matrix

\begin{tabular}{lcccccc}
\hline & MPA & SL & ES & MET & RCC & average \\
\hline MPA & 0.245 & 0.266 & 0.292 & 0.285 & 0.219 & 0.261 \\
SL & 0.061 & 0.066 & 0.048 & 0.047 & 0.087 & 0.061 \\
ES & 0.122 & 0.2 & 0.146 & 0.190 & 0.144 & 0.160 \\
MET & 0.080 & 0.133 & 0.73 & 0.380 & 0.098 & 0.438 \\
RCC & 0.490 & 0.333 & 0.439 & 0.416 & \\
\hline
\end{tabular}

Step5: Multiply the solution matrix with the average of relative percentages matrix

\begin{tabular}{lcccc}
\hline & Events & Publications & New-Registered & Thesis \\
\hline MPA & 0.146 & 0.120 & 0.190 & 0.144 \\
SL & 0.292 & 0.240 & 0.285 & 0.219 \\
ES & 0.073 & 0.079 & 0.095 & 0.109 \\
MET & 0.439 & 0.480 & 0.380 & 0.438 \\
RCC & 0.048 & 0.079 & 0.047 & 0.087 \\
\hline
\end{tabular}


Multiply the solution matrix with the average of relative percentages matrix

\begin{tabular}{llc}
\hline Laboratory & Score & coefficient distribution \\
\hline MPA & 0.211 & $21.1 \%$ \\
SL & 0.120 & $12 \%$ \\
ES & 0.128 & $12.8 \%$ \\
MET & 0.256 & $25.6 \%$ \\
RCC & 0.285 & $28.5 \%$ \\
\hline
\end{tabular}

Based in the result found in the step 5, we conclude that the laboratory RCC is the laboratory that most closely matches the criterion imposed for choosing the ideal laboratory followed by MET, MPA, ES, et SL. The prime objective of this approach is to help managers to improve one or more service areas.

\subsection{TOPSIS:}

Step1: Choose a scale to measure the values of criterion:

The importance of each criterion with respect to another according to the following scale:

$1=$ not interesting at all.

$2=$ not interesting.

$3=$ very uninteresting.

$4=$ moderately interesting.

$5=$ interesting.

$6=$ very interesting.

$7=$ super interesting.

$8=$ perfectly interesting.

Step2: Matrix alternative $\mathrm{X}$ criteria.

\begin{tabular}{lcccc}
\hline & Events & Publications & New-Registered & Thesis \\
\hline MPA & 6 & 5 & 6 & 5 \\
SL & 8 & 8 & 5 & 8 \\
ES & 7 & 6 & 4 & 4 \\
MET & 4 & 7 & 8 & 5 \\
RCC & 5 & 4 & 4 & 4 \\
\hline
\end{tabular}

Allocation of weighting W:

Publication: 0.4

Thesis: 0.3

Events: 0.2

New-Registered: 0.1

Step3: Standardized matrix by criterion (attribute)

We normalize all the scores of the matrix of the levels attributed to the criteria, for that we apply the following formula where $x_{i j}$ criterion:

$$
r_{i j}=\frac{x_{i j}}{\sqrt{\sum_{i=1}^{n} x_{i j}^{2}}}
$$

\begin{tabular}{lcccc}
\hline & Events & Publications & New-Registered & Thesis \\
\hline MPA & 0.43 & 0.36 & 0.43 & 0.41 \\
SL & 0.58 & 0.58 & 0.58 & 0.66 \\
ES & 0.5 & 0.44 & 0.50 & 0.33 \\
MET & 0.29 & 0.50 & 0.29 & 0.413 \\
RCC & 0.36 & 0.29 & 0.36 & 0.33 \\
\hline
\end{tabular}

Step4: Standardized and weighted matrix:

We simply multiply all the entries $r_{i j}$ f the standardized matrix by the weighting associated with each criterion.

$$
r_{i j}=w_{j} \times x_{i j}
$$




\begin{tabular}{lcccc}
\hline & \multicolumn{1}{c}{ Pvents } & Publications & New-Registered & \multicolumn{1}{l}{ Thesis } \\
\hline MPA & 0.086 & 0.144 & 0.043 & 0.123 \\
SL & 0.116 & 0.232 & 0.036 & 0.198 \\
ES & 0.1 & 0.176 & 0.05 & 0.099 \\
MET & 0.058 & 0.2028 & 0.029 & 0.123 \\
RCC & 0.072 & 0.116 & 0.058 & 0.099 \\
\hline
\end{tabular}

Step5: Calculates the ideal favorable solution A+:

For each criterion (attribute) we calculate the most favorable associated value $\mathrm{A}+$ according to the nature of the criterion (favorable or unfavorable).

$$
A^{+}=\left\{\max _{i} x_{i j}\left(i \in J^{+}\right) \mid \min _{i} x_{i j}\left(i \in J^{-}\right)\right\}
$$

\begin{tabular}{ccccc}
\hline & Events & Publications & New-Registered & Thesis \\
\hline MPA & 0.116 & 0.232 & $\mathbf{0 . 0 5 8}$ & 0.198 \\
\hline
\end{tabular}

Step6: Calculates the ideal unfavorable solution A-:

For each criterion (attribute) we calculate the least favorable associated value A- according to the nature of the criterion (favorable or unfavorable).

$$
A^{-}=\left\{\min _{i} x_{i j}\left(i \in J^{+}\right) \mid \max _{i} x_{i j}\left(i \in J^{-}\right)\right\}
$$

\begin{tabular}{lcccc}
\hline & Events & Publications & New-Registered & Thesis \\
\hline MPA & 0.058 & 0.116 & 0.029 & 0.099 \\
\hline
\end{tabular}

At first sight, if one relies solely on the Euclidean distance as a criterion for optimality, this is not enough, because it is the model 'New-Registered 'which is closest to A + and the model 'Publications 'which is the most away from A-. if a single model that meets both criteria would have at this stage and it will represent the best choice. Therefore, it is necessary to determine another metric experiment the two criteria at once called 'proximity factor' defines by the mathematical formula.

Step7: Calculates the deviation of the ideal unfavorable solution from each row of the matrix:

$$
E^{+}, \sqrt{\sum_{j=1}^{m}\left(r_{j}^{+}-r_{i j}\right)^{2}}
$$

\begin{tabular}{ccccc}
\hline & MPA & SL & ES & MET \\
\hline E+ & 0.120 & 0.022 & 0.182 & 0.1033 \\
\hline
\end{tabular}

Step8: Calculates the deviation of the ideal unfavorable solution from each row of the matrix:

$$
E^{-}{ }_{i}=\sqrt{\sum_{j=1}^{m}\left(r_{j}{ }^{-}-r_{i j}\right)^{2}}
$$

\begin{tabular}{cccccc}
\hline & MPA & SL & ES & MET & \\
\hline E- & 0.0483 & 0.1633 & 0.0761 & 0.090 & \\
\hline
\end{tabular}

Step9: Calculates proximity coefficient of the ideal solution and storage in order of choice $\mathrm{S}^{*}$ :

$$
\begin{aligned}
S^{*}{ }_{i} & =\frac{E^{-}{ }_{i}}{E^{-}{ }_{i}+E^{+}{ }_{i}} \\
A^{-} & =\left\{\min _{i} x_{i j}\left(i \in J^{+}\right) \mid \max _{i} x_{i j}\left(i \in J^{-}\right)\right\}
\end{aligned}
$$




\begin{tabular}{lcc}
\hline Laboratory & Score & coefficient distribution \\
\hline MPA & 0.2869 & $13.87 \%$ \\
SL & 0.8536 & $41.25 \%$ \\
ES & 0.2948 & $14.25 \%$ \\
MET & 0.4655 & $22.5 \%$ \\
RCC & 0.1686 & $8.13 \%$ \\
\hline
\end{tabular}

The proximity coefficient of each alternative, as the name suggests, measures the proximity ratio of the most unfavorable ideal solution A- to the most favorable ideal solution. In conclusion the ranking in descending order of the 5 research laboratory models on the basis of the scores and weights provided and the following:

The laboratory RCC is the laboratory that most closely matches the criterion imposed for choosing the ideal laboratory followed by MPA, ES, MET, et SL. The prime objective of this approach is to help managers to improve one or more service areas.

Table 1. Calculated point interval of methods

\begin{tabular}{lccc}
\hline Methods & Minimum point & Maximum point & Mean point \\
\hline AHP & 0.120 & 0.2895 & 0.2 \\
TOPSIS & 0.1686 & 0.8536 & 0.41388 \\
\hline
\end{tabular}

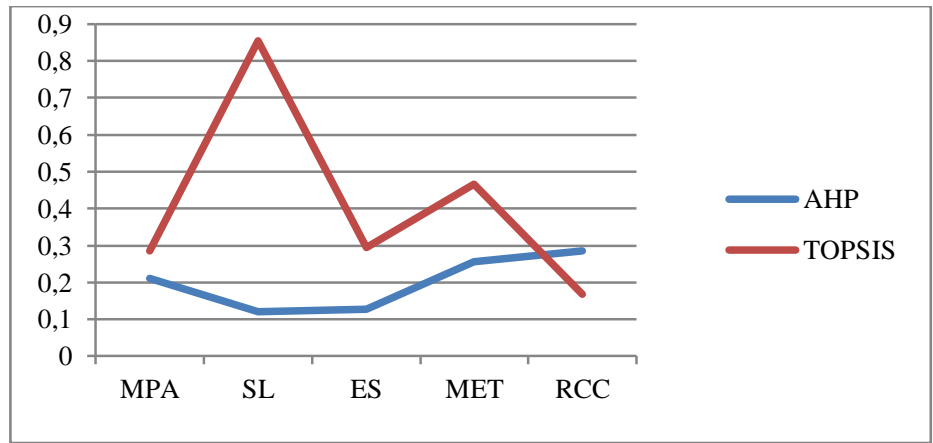

AHP is uniformly worse than TOPSIS [11]. Similarly, AHP and TOPSIS methods are applied to our case with same criteria. Ranking distribution of calculated points are shown in the last table.TOPSIS point interval is higher than others. Moreover as shown, distribution of calculated points with AHP are not distinguishable. TOPSIS is better than AHP because distributions of calculated points with TOPSIS are uniformly distinguishable rather than AHP. Therefore, TOPSIS method has best performance for evaluation.

\section{CONCLUSION}

This paper has addressed the problem of scientific research selection decision making. Decision makers can be able to select the best Laboratory among $\mathrm{N}$ number of alternative laboratory available based on following four criteria's: Events, Publications, New-Registered and Thesis. Each head of the laboratory needs to fill the values for above mentioned criteria's and based on that final data have been assigned to each criterion.

In this paper, we considered one sample numerical example and applied the both methods AHP and TOPSIS in order to calculate weight of each criterion also to select the best laboratory. The proposed technique will help decision makers to select the best laboratory. Numerical results obtained after applying each method gives a final ranking of laboratory and this definitely helps any decision makers to prioritize all available laboratory and choose the better one, which results in his or her bright future.

This work was a real opportunity to present a method of management system of scientific research, using the tool of analytic hierarchy process and TOPSIS. We conclude that TOPSIS minimizes efforts for the decisions-makers to solves many problems and apply governance policy in all respect related to scientific research. 


\section{REFERENCES}

[1] C. Wang, et al.,"Towards a Typology of Adaptive Governance in the Digital Government Context: the Role of Decision-Making and Accountability," Government Information Quarterly, vol. 35, pp. 306-322, Apr 2018.

[2] K. Benmoussa, et al.," Enhanced model for ergonomic evaluation of information systems: application to scientific research information system," International Journal of Electrical and Computer Engineering, vol. 9, pp. 683-694, Feb 2019.

[3] R. J. Martin, et al.," Symbolic-Connectionist Representational Model for Optimizing Decision Making Behavior in Intelligent Systems," International Journal of Electrical and Computer Engineering, Vols 8, pp. 326-332, Feb 2018.

[4] R. Arulmozhiyal and K. Baskaran, "Are Multi-Criteria Decision-Making Tools Useful? An Experimental Comparative Study of Three Methods," European Journal of Operational Research, vol. 264, pp. 462-471, Jan 2018.

[5] M. Aggarwal," Learning of Aggregation Models in Multi Criteria Decision Making," Knowledge-Based Systems, vol. 119, pp. 1-9, Mar 2017.

[6] A. J. Nebro, et al.,"InDM2: Interactive Dynamic Multi-Objective Decision Making Using Evolutionary Algorithms," Swarm and Evolutionary Computation, vol. 40, pp. 184-195, Jun 2018.

[7] R. R. Yager," Categorization in Multi-Criteria Decision Making," Information Sciences, vol. 460-461, pp. 416-423, Sep 2018.

[8] Mustakim, et al.," Eigenvalue of Analytic Hierarchy Process as The Determinant for Class Target on Classification Algorithm," Indonesian Journal of Electrical Engineering and Computer Science, vol. 12, pp. 1257-1264, Dec 2018.

[9] O. S. Vaidya and S. Kumar.,"Analytic hierarchy process: An overview of applications," European Journal of Operational Research, vol. 169, pp. 1-29, Feb 2006.

[10] E. K. Zavadska, et al.," Development of TOPSIS Method to Solve Complicated Decision-Making Problems-An Overview on Developments from 2000 to 2015," International Journal of Information Technology \& Decision Making, vol. 15, pp. 645-682, 2016.

[11] M. İnce, et al.," AHP-TOPSIS Method for Learning Object Metadata Evaluation,"International Journal of Information and Education Technology, vol. 7, pp. 884-887, Dec 2017. 